\title{
Modelling Network Constrained Economic Dispatch Problems
}

\author{
Rodrigo Palma-Benhke, ${ }^{\ddagger}$ Senior Member, IEEE, Andy Philpott ${ }^{\dagger}$ Member, IEEE, Alejandro Jofré $`$ and Marcelo \\ Cortés-Carmona ${ }^{\sharp}$, Graduate Student, IEEE,
}

\begin{abstract}
The behaviour of DC Load-flow formulations when they are used in economic dispatch and nodal pricing models is discussed. It is demonstrated that non-negative prices in these models are sufficient to guarantee global optimality of any local optimum, even if the feasible region is not convex, and so a negative nodal price is an indicator of a possible loss in optimality. It is also discuss the possible effect that negative prices might have on algorithms that assume this convexity.
\end{abstract}

Index Terms-Convex Optimization, Economic Dispatch, Quadratic Programming, Linear Programming.

\section{INTRODUCTION}

In power markets there is an increasing need for improving the representation of high-voltage transmission networks in order to better support market design alternatives, priceformation mechanisms, and for general operation and planning decisions. In most cases, this process involves the definition of more complex mathematical models. Different optimization approaches based on DC Load-flow formulations are extensively used in this field [1]-[4].

In this paper we study the behaviour of DC Load-flow formulations when used in economic dispatch and nodal pricing models. Our focus is on instances of these models in which negative prices are observed at some nodes. A negative price at a node indicates a situation in which the system cost can be reduced by more consumption of power at the node. When there is a free disposal of power at this node, a simple economic argument shows that the nodal price must be nonnegative. So negative prices can only occur when flow balance constraints in the dispatch model are modeled as equations.

When the DC-load flow model does not include line losses, it is a linear program, which is easily solved using standard software [5], [6]. Linear programs are convex optimization problems, and enjoy all their desirable properties. Although negative prices may occur in these models, their existence need not be a matter of any concern. On the other hand, when losses are modeled as quadratic functions of the line flow, it is well known that (without free disposal at the nodes) the feasible

\footnotetext{
¥ Department of Electrical Engineering, University of Chile, Avenida Tupper 2007, Santiago, Chile - Tel: 56-2- 9784203 - email: rodpalma@cec.uchile.cl.

$\dagger$ Electric Power Optimization Centre, Department of Engineering Science, University of Auckland, - email: a.philpott@auckland.ac.nz.

- Center of Mathematical Modelling, University of Chile, Avenida Blanco Encalada 2120, Santiago, Chile - Telephone: 56-2-9784454 - email: ajofre@dim.uchile.cl.

\# Department of Electrical Engineering, University of Chile and University of Antofagasta, Avenida Tupper 2007, Santiago, Chile, email: mcortes@uantof.cl.
}

region of the dispatch model is no longer convex [7]. This means that the benefits of convex optimization are no longer guaranteed.

In this paper we investigate the extent to which this loss of convexity is material in solving realistic dispatch problems. We demonstrate that non-negative prices are sufficient to guarantee global optimality, even if the feasible region is not convex, and so a negative nodal price is an indicator of a possible problem for optimization software. In particular, we show that linear programming software that approximates losses by piecewise linear functions will not represent losses properly as it becomes more accurate. Quadratic programming software that assumes positive definite Hessian matrices may also encounter problems in solving such problems. Finally nonlinear optimization software that yields at best a local optimum may not give a global optimal solution when prices are negative.

The paper is laid out as follows. In the next section, we give some general mathematical results that can be applied to the dispatch problem. A general mathematical formulation of the economic dispatch problem is then presented in Section III, and we present a three-busbar model to illustrate the formulation, and to serve as an example of some of the difficulties we describe. We first show in Section IV how linear programming software might fail when applied to this problem. In Section V we compute the Hessian matrix of the Lagrangian with respect to the flow-balance constraints, and show how this might fail to be positive semi-definite with negative prices. In Section VI a nonconvex six-busbar example is described and used to illustrate how a negative price might be an indicator of a local optimum solution. Finally, Section VII presents the conclusions of the work.

\section{PRELIMINARIES}

Consider the general optimization problem

$$
\begin{aligned}
& E(u): \min \sum_{i} h_{i}\left(q_{i}\right) \\
& \text { s.t. } g_{i}(f)+q_{i}=u_{i}, \quad i=1,2, \ldots, n \\
& A f+B q \quad=\quad b \text {, } \\
& f \in F, \quad q \in Q \text {. }
\end{aligned}
$$

where each $h_{i}$ is a convex function and each $g_{i}$ is a concave function; $A$ and $B$ are $p \times m$ and $p \times n$ real matrices; $b$ lies in $R^{p}$; and $F$ and $Q$ are convex sets in $R^{m}$ and $R^{n}$ respectively. 
We define the following relaxation of $E(u)$ :

$$
\begin{aligned}
& G(u): \min \sum_{i} h_{i}\left(q_{i}\right) \\
& \text { s.t. } g_{i}(f)+q_{i} \geq u_{i}, \quad i=1,2, \ldots, n \\
& A f+B q=b \text {, } \\
& f \in F, \quad q \in Q \text {. }
\end{aligned}
$$

Assume that every feasible point of $E(u)$ and $G(u)$ satisies a constraint qualification (see e.g. [8]). Let $\lambda_{i}(E), i=$ $1,2, \ldots, n$, be the Lagrange multipliers from the first set of constraints for $E$ (and let $\lambda_{i}(G)$ be defined similarly).

Proposition 1: $\lambda_{i}(G) \geq 0, i=1,2, \ldots, n$.

Proof: See [8].

Proposition 2: If $\lambda_{i}(E) \geq 0, i=1,2, \ldots, n$ for a locally optimal solution to $E(u)$, then this is also optimal for $G(u)$.

Proof: Suppose that $\left(q^{*}, f^{*}\right)$ solves $E(u)$, with $\lambda_{i}(E) \geq$ $0, i=1,2, \ldots, n$. Then $\left(q^{*}, f^{*}, \lambda(E)\right)$ satisfies the KarushKuhn-Tucker conditions of $G(u)$. Since $G(u)$ is a convex programming problem these conditions are sufficient.

Corollary 3: A locally optimal solution to $E(u)$ with $\lambda_{i}(E) \geq 0, i=1,2, \ldots, n$ is also globally optimal.

Corollary 4: Let $\phi_{E}(u)$ be the optimal value of $E(u)$. If $\lambda_{i}(E(u)) \geq 0, i=1,2, \ldots, n$ for every $u$ then $\phi_{E}(u)$ is convex.

Proof: Follows from the convexity of the optimal value of the convex programming problem $G(u)$ (see [8]).

\section{The ECONOMIC Dispatch PROBLEM}

\section{A. The General Case}

The economic dispatch problem for an electricity generation and transmission system is typically formulated by expressing link flows and losses in terms of voltage angles at each bus [2].

$$
\begin{aligned}
Z= & \operatorname{Min}\left\{\sum_{i=1}^{N G} C_{G i}\left(P_{G i}\right)+\sum_{i=1}^{N D} C_{U i}\left(P_{U i}\right)\right\} \\
& \text { s.t. } \\
& \sum_{k \in \Omega_{i}^{G}} P_{G_{k}}-\sum_{j \in \Omega_{i}^{N}}\left(\frac{\theta_{i}-\theta_{j}}{x_{i j}}+\frac{r_{i j}\left(\theta_{i}-\theta_{j}\right)^{2}}{2 x_{i j}^{2}}\right)+ \\
& \sum_{k \in \Omega_{i}^{C}} P_{U_{k}}=\sum_{k \in \Omega_{i}^{C}} P_{C_{k}} \forall i \in N N \\
& \theta_{i}-\theta_{j} \leq x_{i j} \bar{F}_{i j} \forall(i, j) \in \Omega^{N N} \\
& \theta_{j}-\theta_{i} \leq x_{i j} \bar{F}_{j i} \\
& \mathbf{x}_{E} \leq \mathbf{x}_{E} \leq \overline{\mathbf{x}}_{E}
\end{aligned}
$$

where:

- $N G=$ number of generators

- $N D=$ number of demands

- $N N=$ number of busbars

- $\Omega_{i}^{N}=$ set of nodes adjacent to node $i$

- $\Omega_{i}^{G}=$ set of generators at node $i$

- $\Omega_{i}^{C}=$ set of demands at node $i$

- $C_{G i}=$ convex generation cost function,

- $C_{U i}=$ convex cost function of unserved energy.

- $P_{G i}=$ generation active power injection in per unit.

- $P_{U i}=$ unserved energy (active power) in per unit.
- $\theta_{i}=$ voltage phase angle from node $i$ expressed in radians.

- $x_{i j}=$ line series reactance expressed in per unit.

- $r_{i j}=$ represents the equivalent resistance expressed in per unit where $r r_{i j}$ is line series resistance expressed in per unit.

- $P_{C_{j}}=$ active power load expressed in per unit.

- $P_{i j}=$ active power flow between nodes $i$ and $j$ expressed in per unit.

- $\bar{F}_{i j}=$ maximum active power flow on transmission line between nodes $i$ and $j$ expressed in per unit.

- $\mathbf{x}_{E}=\left[\begin{array}{lll}P_{G} & P_{U} & \theta\end{array}\right]=$ vector of all optimization variables.

Ohmic losses $P L_{i j}$ for each transmission line can be represented [1] by the nonlinear function

$$
P L_{i j}=2 \frac{r r_{i j}}{r r_{i j}^{2}+x_{i j}^{2}}\left(1-\cos \left(\theta_{i}-\theta_{j}\right)\right)
$$

that has a second-order approximation given by the quadratic function

$$
P L_{i j} \approx \frac{r r_{i j}}{r r_{i j}^{2}+x_{i j}^{2}}\left(\theta_{i}-\theta_{j}\right)^{2},
$$

which on substitution of equivalent resistance $r_{i j}=\frac{r r_{i j} x_{i j}^{2}}{r r_{i j}^{2}+x_{i j}^{2}}$ gives

$$
P L_{i j} \approx \frac{r_{i j}\left(\theta_{i}-\theta_{j}\right)^{2}}{x_{i j}^{2}} .
$$

This can be further simplified using the fact that $r r_{i j} \ll x_{i j}$ $\left(r r_{i j}=0.25 x_{i j}\right.$ or less) to give

$$
P L_{i j} \approx r r_{i j} P_{i j}^{2}=\frac{r r_{i j}}{x_{i j}^{2}}\left(\theta_{i}-\theta_{j}\right)^{2} .
$$

The resulting economic dispatch problem has convex cost function, quadratic equality constraints (node balances), linear inequality constraints and bounds for each variable. It is easy to see by making the substitution $f_{i j}=\frac{\theta_{i}-\theta_{j}}{x_{i j}}, i<j$, that the economic dispatch problem is in an equivalent form to $E(u)$. Here the function $g_{i}(f)$ takes the form of $\sum_{j<i}\left(f_{j i}-\right.$ $\left.\frac{r_{i j}}{2} f_{j i}^{2}\right)+\sum_{i<j}\left(-f_{i j}-\frac{r_{i j}}{2} f_{i j}^{2}\right)$. It is important to note that the economic dispatch problem can also be modeled with nonconvex generator cost curves [9], a case that falls outside the setting we discuss here.

\section{B. Example 3-node system}

To motivate our discussion we shall study the realistic 220 $\mathrm{kV}$ three-busbar system shown in Fig. 1. This case study is based on realistic data and can be interpreted as a subnetwork of more extended power system. Here, for each line we consider reactances $x_{i j}=0.4 \Omega / \mathrm{km}$ and equivalent resistances $r_{i j}=0.04 \Omega / k m \rightarrow r r_{i j}=0.040408 \Omega / k m$. Using a reference power $\mathrm{Sb}=100 \mathrm{MVA}$, the resulting reactances and resistances in per unit are shown in Fig. 1.

- Line lengths: Line $\mathrm{A}=$ Line $\mathrm{B}=121 \mathrm{~km}$, Line $\mathrm{C}=181.5$ $\mathrm{km}$.

- The transmission capacity of Line $\mathrm{C}$ expressed in active power is set to $F_{23}=50 \mathrm{MW}$. 


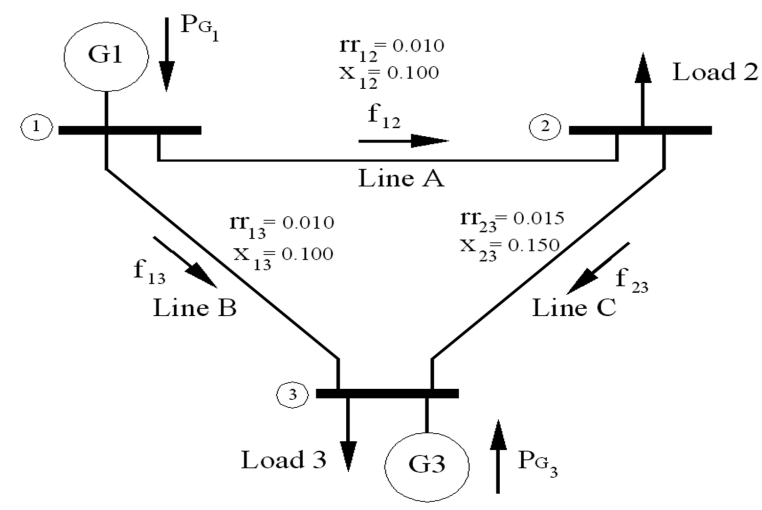

Fig. 1. Three busbar system

- Generation costs: Reservoir $=$ Gen $1 \rightarrow C_{1}=1 \$ / M W h$ (strategic value of stored water), Gen $3 \rightarrow C_{3}=50$ $\$ / M W h$.

- Load $2=10 M W$ and Load $3=200 M W$.

- Unserved energy costs of $500 \$ / M W h$ for each load.

The optimal economic dispatch for this example can be computed using nonlinear programming software (e.g. MINOS [6]) and summarized as follows:

- $P_{G 1}^{*}=196.84 \mathrm{MW}, P_{G 3}^{*}=15.73 \mathrm{MW}$.

- Total Losses $=2.57 M W \rightarrow 1.22 \%$.

- System costs $=983.5 \$ / h$.

- $\lambda_{1}=1 \$ / M W h, \lambda_{2}=-47.591 \$ / M W h, \lambda_{3}=50$ $\$ / M W h$.

The Lagrange multipliers $\lambda_{i}$ relate to the node-balance constraints and give the nodal prices at optimality, one of which $\lambda_{2}$ is negative. The software can be shown to terminate at a locally optimal solution, but since $\lambda_{2}<0$, we have no guarantee that this is a globally optimal solution, although this can be verified in this example by exhaustively checking the Karush-Kuhn-Tucker conditions. Recall that optimization problems of the form $E(u)$ are not convex programming problems. In the next section it is shown that further analysis is needed to define global optimality.

\section{Piecewise Linear Approximation of Losses}

As shown in Proposition 2, if a locally optimal solution to the economic dispatch problem has non-negative Lagrange multipliers at optimality, then this solution is also a (global) solution to the (convex) inequality constrained problem, and so it is a global solution to the dispatch problem. The fact that the solution is also a solution to $G(u)$ means that we may approximate $G(u)$ by a linear program (say $L(u)$ ) and expect that the solution to $L(u)$ is close to the global optimum of $G(u)$ and hence $E(u)$. Many economic dispatch systems (see e.g. [10]) use linear programming in this way.

The linear programming approximation of losses can fail when the optimal solution to $E(u)$ has a negative Lagrange multiplier as in our example (this fact is well known in the load-flow modelling community, see e.g. [11]). To illustrate this we solved the three-busbar example using following step

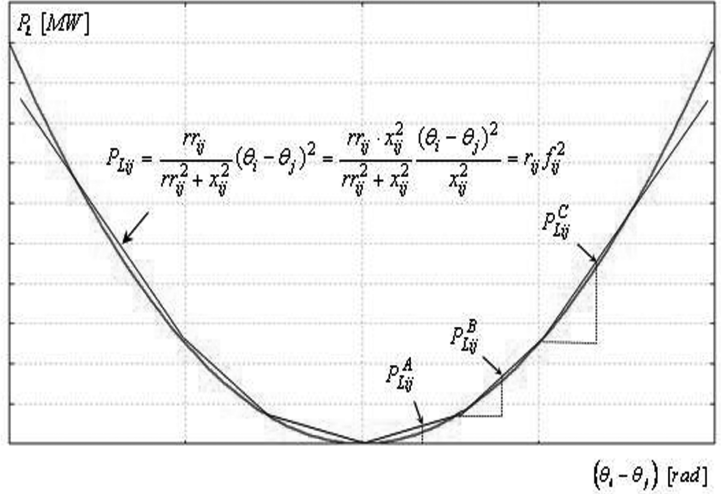

Fig. 2. Ohmic losses representation

piece-wise linear loss functions:

$$
P_{L i j}=\sum_{k=A}^{C} m_{i j}^{k}\left(\Delta \theta_{i j}^{k}\right) ; \quad \theta_{i}-\theta_{j}=\sum_{k=A}^{C} \Delta \theta_{i j}^{k} .
$$

Considering three step piece-wise linear, that is, $k=A, B, C$, the loss model in the three-busbar system can be writen as

$$
\begin{aligned}
& P_{L 12}=100\left(\theta_{1}-\theta_{2}\right)^{2} \approx P_{L 12}^{A}+P_{L 12}^{B}+P_{L 12}^{C} \\
& P_{L 13}=100\left(\theta_{1}-\theta_{3}\right)^{2} \approx P_{L 13}^{A}+P_{L 13}^{B}+P_{L 13}^{C} \\
& P_{L 23}=66 . \overline{6}\left(\theta_{2}-\theta_{3}\right)^{2} \approx P_{L 23}^{A}+P_{L 23}^{B}+P_{L 23}^{C}
\end{aligned}
$$

Fig. 2 shows the ohmic losses representation by three linear functions.

Solving the resulting optimization problem for three loss function levels in each transmission line we obtained the following loss values in $\mathrm{MW}$ :

$$
\begin{array}{lcc}
P_{L 12}^{A}=0.0000 & P_{L 12}^{B}=0.2709 & P_{L 12}^{C}=0.9863 \\
P_{L 13}^{A}=0.1306 & P_{L 13}^{B}=0.745 & P_{L 13}^{C}=0.9679 \\
P_{L 23}^{A}=0.0862 & P_{L 23}^{B}=0.3065 & P_{L 23}^{C}=0.0000
\end{array}
$$

Fig. 3 shows the optimal result achieved for the ohmic losses in Line A (between nodes 1 and 2).

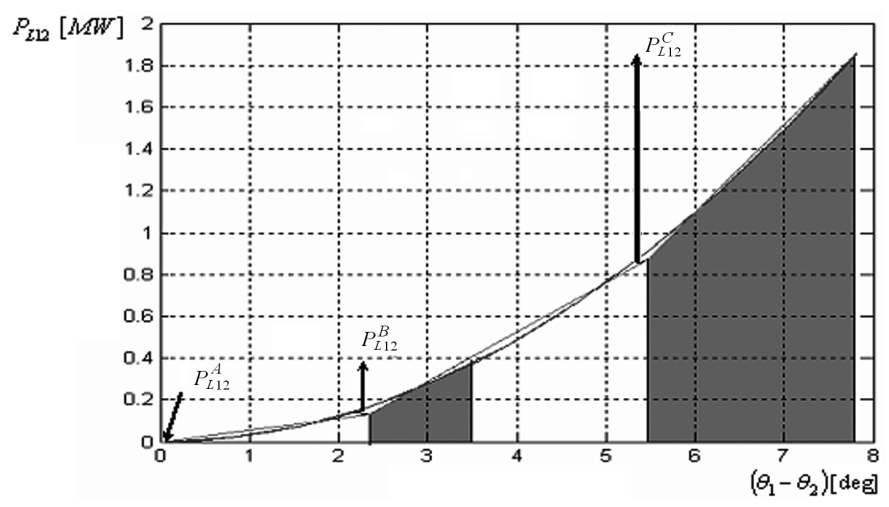

Fig. 3. Ohmic losses in line A with linear functions

It can be observed that the optimization arrives at an infeasible solution from the physical point of view. The software tries to maximize losses in Line A using the second and third level of the loss function $P_{L 12}^{B}, P_{L 12}^{C}$. The correct physical losses 
are $0.3698 M W$, while the ohmic losses based on the linear approximation are $1.2572 \mathrm{MW}$. In this way, more power can be allocated by the cheaper generation at busbar one. So the piecewise linear approximation has failed to represent the problem in the way we intended.

We might have expected some problems here since we are approximating a nonconvex optimization problem with a convex one. Indeed the example has a negative price so we do not have a guarantee that $E(u)$ has the same solution as $G(u)$ its convexification. We proceed to show under fairly mild conditions on the optimal solution that a negative price at any node indicates that a linear programming model of the form above will give an incorrect flow representation if there are enough pieces in the piecewise linear representation of the loss curves.

Our result is stated in the framework of the problem $E(u)$, for a transmission network of directed lines $i j, i<j$, where

$$
g_{i}(f)=\sum_{j<i}\left(f_{j i}-\frac{r_{j i}}{2} f_{j i}^{2}\right)+\sum_{j>i}\left(-f_{i j}-\frac{r_{i j}}{2} f_{i j}^{2}\right),
$$

and for each transmission line $i j,-F_{i j}<f_{i j}<F_{i j}$. We assume that $r_{i j}>0$ for all lines $i j$ implying that $\frac{r_{i j}}{2} f_{i j}^{2}$ is a strictly convex function. In the linear program, we model $l_{i j}=\frac{r_{i j}}{2} f_{i j}^{2}$ as piecewise linear convex functions, where

$$
\begin{array}{ll}
f_{i j}^{\nu}=-F_{i j}+y_{i j}^{1}+y_{i j}^{2}+\ldots+y_{i j}^{\nu}, & i<j, \\
0 \leq y_{i j}^{k} \leq b_{i j}^{k}, k=1,2, \ldots, \nu, & i<j, \\
l_{i j}^{\nu}=\frac{r_{i j}}{2} F_{i j}^{2}+m_{i j}^{1} y_{i j}^{1}+m_{i j}^{2} y_{i j}^{2}+\ldots+m_{i j}^{\nu} y_{i j}^{\nu}, & i<j,
\end{array}
$$

where the strict convexity assumption gives increasing slopes, i.e.

$$
m_{i j}^{k}<m_{i j}^{k+1},
$$

and each slope is in the interval $(-1,1)$, since the marginal loss can never exceed the marginal flow.

Our result requires the following definition.

Definition 5: A dispatch is degenerate if there exists a node $i$, at which every generator is either not dispatched or fully dispatched, and all lines $i j$ have flow either at zero or an upper bound or a lower bound. (The dispatch computed in the previous 3-node example is not degenerate.)

Proposition 6: Suppose the optimal dispatch is not degenerate. Any node $i$ with a negative price in the optimal dispatch has $q_{k}=0, k \in \Omega_{i}^{G}$ and at least one incident line with nonzero flow between its bounds.

Proof: Consider node $i$ with a negative price $\lambda_{i}<0$ for some optimal dispatch. Since the dispatch is optimal, and generator costs are non-negative, the Karush-Kuhn-Tucker conditions imply that every generator at node $i$ is dispatched zero. Since the dispatch is not degenerate, then there is some line $i j$ with $-F_{i j}<f_{i j}<F_{i j}$ and $f_{i j} \neq 0$, or some line $j i$ with $-F_{j i}<f_{j i}<F_{j i}$ and $f_{j i} \neq 0$, i.e. $i$ has at least one incident line with nonzero flow between its bounds.

In our example, the line A connecting buses 1 and 2 has power flowing into node 2 at a rate less than the line's capacity, as predicted by the proposition. If demand at each node is nonnegative, then Proposition 6 implies that any node $i$ with a negative price in a nondegenerate optimal solution has some positive flow entering $i$ from some other node $j$. (Otherwise all line flows would be directed away from $i$ which would be feasible only of demand was negative.) Given such a solution, we can rename the node indices so that $0<f_{j i}<F_{j i}$. This allows us to show the following result.

Proposition 7: Suppose demand at each node is nonnegative, the optimal dispatch $(q, f)$ for $E(u)$ is nondegenerate, and some node has a negative price. Suppose $L P^{\nu}$ is a sequence of linear programming problems with a piecewise linear loss function for each line converging uniformly to the corresponding quadratic loss function. Suppose for all $\nu$ the optimal solution $\left(q^{\nu}, f^{\nu}, \lambda^{\nu}\right)$ to $L P^{\nu}$ correctly represents the losses. Then $\left(q^{\nu}, f^{\nu}, \lambda^{\nu}\right)$ does not converge to $(q, f, \lambda)$.

Proof: Let $i$ be the node with a negative price in the optimal solution. By proposition 6 and the discussion above there is no generation at $i$, and at least one line $j i$ with $0<f_{j i}<F_{j i}$. Suppose $\left(q^{\nu}, f^{\nu}, \lambda^{\nu}\right) \rightarrow(q, f, \lambda)$. Then for sufficiently large $\nu$ we have $\lambda_{i}^{\nu}<0$ and

$$
0<f_{j i}^{\nu}<F_{j i} .
$$

The flow

$$
f_{i j}^{\nu}=-F_{i j}+y_{i j}^{1}+y_{i j}^{2}+\ldots+y_{i j}^{\nu}
$$

is the sum of variables $y_{j i}^{k}$ from $\nu$ loss sections, and by assumption $y_{j i}^{k}=b_{j i}^{k}$ for all sections up to the one corresponding to $f_{j i}^{\nu}$, and $y_{j i}^{k}=0$ for the sections beyond. (We can make $\nu$ large enough so the section $k$ corresponding to $f_{j i}^{\nu}$ is between 2 and $\nu-1$.)

Let $\rho^{k}$ be the reduced cost of each of these variables at optimality of the problem $L P^{\nu}$. Then it is easy to show that

$$
\rho^{k}=\left(\lambda_{i}^{\nu}+\lambda_{j}^{\nu}\right) m_{j i}^{k}+\left(\lambda_{j}^{\nu}-\lambda_{i}^{\nu}\right)
$$

where $m^{k}$ is the slope for section $k$. We have

$$
\left(\lambda_{j}^{\nu}-\lambda_{i}^{\nu}\right)>\left(\lambda_{j}^{\nu}+\lambda_{i}^{\nu}\right)
$$

so if $\left(\lambda_{j}^{\nu}+\lambda_{i}^{\nu}\right) \geq 0$, then $\rho_{k}>0$ for all $k$, and so $f_{j i}^{\nu}=-F_{j i}$, yielding a contradiction. Thus we have

$$
\lambda_{j}^{\nu}+\lambda_{i}^{\nu}<0 .
$$

Since $0<f_{j i}^{\nu}<F_{j i}$, we have $0<m_{k}<m_{k+1}$ which implies that

$$
\begin{aligned}
\rho_{k} & =\left(\lambda_{i}^{\nu}+\lambda_{j}^{\nu}\right) m_{j i}^{k}+\left(\lambda_{j}^{\nu}-\lambda_{i}^{\nu}\right) \\
& >\left(\lambda_{i}^{\nu}+\lambda_{j}^{\nu}\right) m_{j i}^{k}+\left(\lambda_{j}^{\nu}-\lambda_{i}^{\nu}\right) \\
& =\rho_{k+1}
\end{aligned}
$$

This inequality contradicts the assumption that $\left(q^{\nu}, f^{\nu}, \lambda^{\nu}\right)$ solves $L P^{\nu}$, as it entails that either $y_{j i}^{1}=b_{j i}^{1}$ and $\rho_{1}>0$, or $y_{j i}^{\nu}=b_{j i}^{\nu}$ and $\rho_{\nu}<0$.

\section{QUADRATIC OPTIMIZATION OF LINE LOSSES}

The failure of linear programming in these circumstances points to the use of optimization software that will compute optimal solutions with quadratic functions. The quadratic terms from the losses can be placed in a Lagrangian, where the Lagrange multipliers $\lambda_{i}$ are chosen to be those that pertain at the global optimal solution (We shall assume regularity 
conditions that ensure these exist). The Lagrangian for the economic dispatch problem can then be expressed as

$$
\begin{aligned}
\mathcal{L}\left(P_{G}, P_{U}, \theta\right)= & \sum_{i=1}^{N G} C_{G i}\left(P_{G i}\right)+\sum_{i=1}^{N D} C_{U i}\left(P_{U i}\right) \\
& +\sum_{i=1}^{N N} \lambda_{i}\left[\sum_{j \in \Omega_{i}^{C}} P_{C_{j}}-\sum_{j \in \Omega_{i}^{G}} P_{G_{j}}+\ldots\right. \\
& \sum_{j \in \Omega_{i}^{N}}\left(\frac{\theta_{i}-\theta_{j}}{x_{i j}}+\frac{r_{i j}\left(\theta_{i}-\theta_{j}\right)^{2}}{2 x_{i j}^{2}}\right)- \\
& \left.\sum_{j \in \Omega_{i}^{C}} P_{U i}\right]
\end{aligned}
$$

It can be observed that for positive multipliers $\lambda$, the Lagrangian is convex in $P_{G}, P_{U}$, and $\theta$. For linear/quadratic functions $C_{G i}(\cdot)$ and $C_{U i}(\cdot), \mathcal{L}\left(P_{G}, P_{U}, \theta\right)$ is easily seen then to be a positive semi-definite quadratic form.

If some Lagrange multiplier $\lambda_{i}$ is negative, then $\mathcal{L}\left(P_{G}, P_{U}, \theta\right)$ may not be positive semi-definite. To investigate this we will compute its explicit form. Let $A$ be the adjacency matrix of the network

$$
a_{i j}=\left\{\begin{array}{cc}
1 & \text { bus } i \text { and } j \text { are adjacent } \\
0 & \text { otherwise }
\end{array}\right.
$$

$$
\begin{aligned}
\mathcal{L}\left(P_{G}, P_{U}, \theta\right)= & \sum_{i=1}^{N N} \lambda_{i} \sum_{j=1}^{N N} a_{i j}\left(\frac{\theta_{i}-\theta_{j}}{x_{i j}}+\frac{r_{i j}\left(\theta_{i}-\theta_{j}\right)^{2}}{2 x_{i j}^{2}}\right) . . \\
= & \sum_{i=1}^{N N} \sum_{j=1}^{N N} \lambda_{i} a_{i j}\left(\frac{\theta_{i}-\theta_{j}}{x_{i j}}+\frac{r_{i j}\left(\theta_{i}-\theta_{j}\right)^{2}}{2 x_{i j}^{2}}\right) . . \\
= & \sum_{i=1}^{N N} \sum_{j=1}^{N N} \lambda_{i} a_{i j} \frac{\theta_{i}-\theta_{j}}{x_{i j}}+ \\
& +\sum_{i=1}^{N N} \sum_{j=1}^{N N} \lambda_{i} a_{i j} \frac{r_{i j}\left(\theta_{i}-\theta_{j}\right)^{2}}{2 x_{i j}^{2}}+. .
\end{aligned}
$$

The Hessian $H$ of the Lagrangian is defined by

$$
\begin{aligned}
H_{i i} & =\sum_{j=1}^{N N} \lambda_{i} a_{i j} \frac{r_{i j}}{x_{i j}^{2}}+\sum_{j=1}^{N N} \lambda_{j} a_{j i} \frac{r_{j i}}{x_{j i}^{2}} \\
H_{i j} & =-\lambda_{i} a_{i j} \frac{r_{i j}}{x_{i j}^{2}}-\lambda_{j} a_{j i} \frac{r_{j i}}{x_{j i}^{2}}
\end{aligned}
$$

Let $\sigma_{i j}=\sigma_{j i}=a_{i j} \frac{r_{i j}}{x_{i j}^{2}}$. Then

$$
\begin{aligned}
H_{i i} & =\sum_{j=1}^{N N}\left(\lambda_{i}+\lambda_{j}\right) \sigma_{i j} \\
H_{i j} & =-\left(\lambda_{i}+\lambda_{j}\right) \sigma_{i j}
\end{aligned}
$$

Observe that

$$
\sum_{j} H_{i j}=\sum_{i} H_{i j}=0
$$

and so $H$ is singular. Also observe that

$$
\begin{aligned}
\sum_{i \neq k} \sum_{j \neq k} H_{i j}= & \sum_{i \neq k} \sum_{l=1}^{N N}\left(\lambda_{i}+\lambda_{l}\right) \sigma_{i l}+ \\
& \sum_{i \neq k} \sum_{j \neq k}-\left(\lambda_{i}+\lambda_{j}\right) \sigma_{i j} \\
= & \sum_{i \neq k}\left(\lambda_{i}+\lambda_{k}\right) \sigma_{i k}=H_{k k}
\end{aligned}
$$

In the three-bus example

$$
\left(\sigma_{i j}\right)=\left[\begin{array}{ccc}
0 & 1 & 1 \\
1 & 0 & \frac{2}{3} \\
1 & \frac{2}{3} & 0
\end{array}\right]
$$

SO

$$
H=100\left[\begin{array}{ccc}
2 \lambda_{1}+\lambda_{2}+\lambda_{3} & -\lambda_{1}-\lambda_{2} & -\lambda_{1}-\lambda_{3} \\
-\lambda_{1}-\lambda_{2} & \lambda_{1}+\frac{5}{3} \lambda_{2}+\frac{2}{3} \lambda_{3} & -\frac{2}{3}\left(\lambda_{2}+\lambda_{3}\right) \\
-\lambda_{1}-\lambda_{3} & -\frac{2}{3}\left(\lambda_{2}+\lambda_{3}\right) & \lambda_{1}+\frac{2}{3} \lambda_{2}+\frac{5}{3} \lambda_{3}
\end{array}\right]
$$

Recall that $\lambda_{1}=1, \lambda_{2}=-47.591, \lambda_{3}=50$, so $\lambda_{1}+\frac{5}{3} \lambda_{2}+$ $\frac{2}{3} \lambda_{3}<0$, which means that $H$ is indefinite for these choices of $\lambda$. This will cause problems for quadratic programming solvers (e.g. some interior point methods) that require at least positive semi-definite Hessian matrices. It is interesting to observe, however, for this example that $H$ restricted to the tangent plane of the active line capacity constraint

$$
100\left(\frac{\theta_{2}-\theta_{3}}{0.15}\right)=50
$$

gives a reduced Hessian

$$
H_{r}=100\left[\begin{array}{cc}
2 \lambda_{1}+\lambda_{2}+\lambda_{3} & -\left(2 \lambda_{1}+\lambda_{2}+\lambda_{3}\right) \\
-\left(2 \lambda_{1}+\lambda_{2}+\lambda_{3}\right) & 2 \lambda_{1}+\lambda_{2}+\lambda_{3}
\end{array}\right]
$$

that is positive semi-definite as long as $2 \lambda_{1}+\lambda_{2}+\lambda_{3} \geq 0$. Thus a reduced gradient algorithm that identified this active constraint would not have to deal with an indefinite Lagrangian. (The solution computed in section III was found using the reduced gradient nonlinear optimization package MINOS [6]).

\section{Nonconvex SiX-Busbar Example}

In Section IV it was demonstrated that a negative price leads to the failure of linear programming approximations of the dispatch model, that to some extent is overcome by nonlinear programming algorithms. Of course the problem $E(u)$ is not convex, and so we have no guarantee that the nonlinear programming system will locate the global optimum. To illustrate this, consider two identical power exchanges linked by a transmission line with ohmic losses in nodes with negative marginal prices. For this analysis we used twice the previous three busbar example interconnecting both systems at their respective busbar 2 (see Fig. 4).

The optimal economic dispatch after they are connected is not symmetric. In order to allocate more power from the cheap generation at generator $G 1$, energy is transferred from the upper to the lower system $(15.85 M W)$. This yields an increase of load at Busbar 2 that reduces the dispatch of the expensive generator $G 3$ to zero. In the lower system we observe the opposite behaviour. Nevertheless, the final 


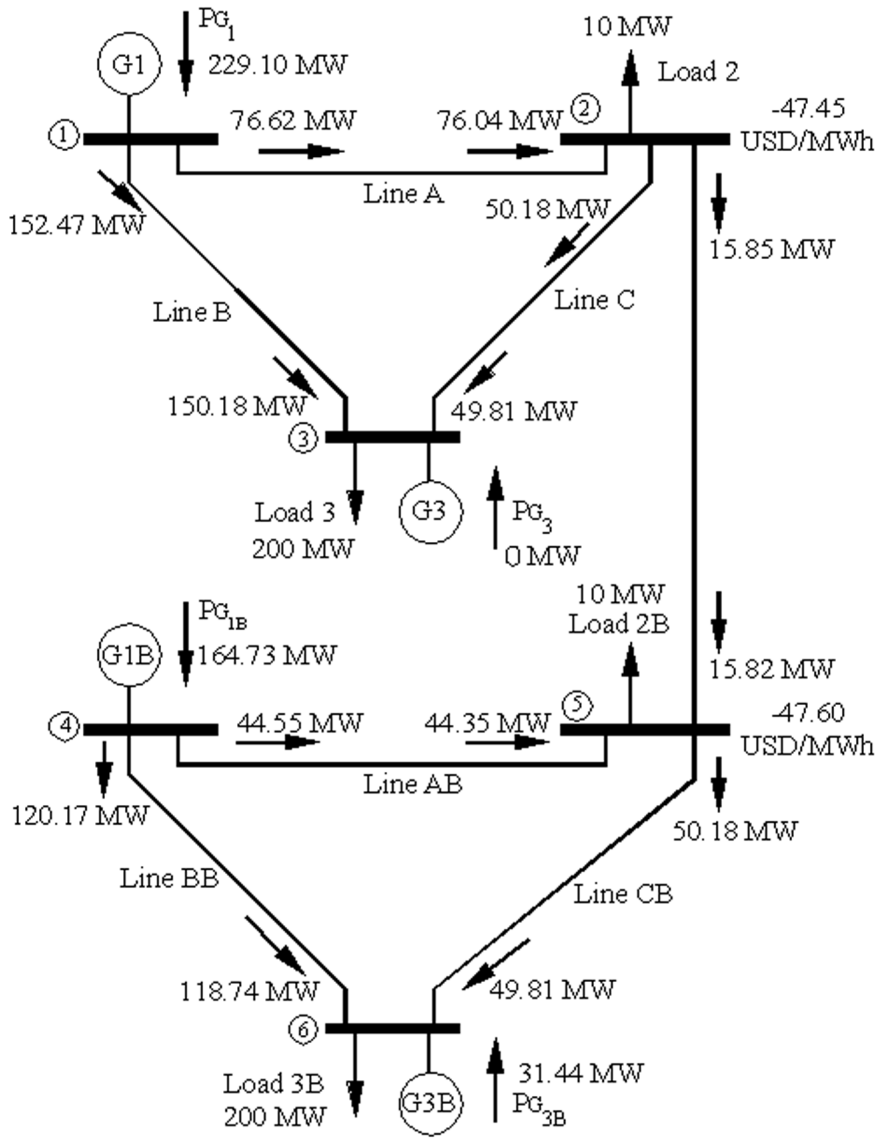

Fig. 4. Two symetrical systems/markets

result is cheaper than twice the costs of the operation of two disconnected networks $(2 * 983.5=1967.0>1965.99 \$ / h)$. When given a starting point equal to the optimal flow in each separate system, and no flow in the connecting line, MINOS terminates at this local optimal solution.

\section{CONCLUSIONS}

In this paper we have discussed the behaviour of DC Load-flow models with ohmic losses when they are used in transmission constrained economic dispatch and nodal pricing models. We have shown that negative nodal prices at the optimal dispatch solution can indicate problems for optimization software. A negative nodal price at a non degenerate solution means that linear programming with piecewise linear losses will fail to converge as the loss representation becomes more accurate. Moreover in complicated transmission networks, a negative price can indicate that only a local optimal solution has been found. In many cases however negative nodal prices present no problems for commercial nonlinear programming codes.

\section{ACKNOWLEDGEMENTS}

We would like to acknowledge the financial support provided by Fondecyt Grant \#1080668 and the Royal Society of New Zealand International Science and Technology Linkages
Grant ISATB04-I09. We want to thank Mr Tony Downward, Mr. Ignacio Alarcón and Mr. Rigoberto Torres for fruitful discussions on this topic.

\section{REFERENCES}

[1] M. River, I. J. Pérez-Arriaga and G. Luengo, "A model for computation of spot prices in interconnected power systems," presented at the 10th PSCC Conf., Graz, Austria, 1990.

[2] A. J.Wood and B. F. Wollenberg, Power Generation, Operation and Control. New York: Wiley, 1996.

[3] A. B. Philpott , "Experiments with load flow pricing models", presented at the CRNEC Policy Conference, Auckland, New Zealand, 1999.

[4] B. Stott, J. Jardin J. and O. Alsac, "DC power flow revisited," IEEE Trans. Power Syst., vol. 24 , pp. 1290-1300, Aug. 2009.

[5] Ilog, "CPLEX 8.1 Reference Manual", 2002.

[6] B. A. Murtagh and M. A. Saunders, "MINOS 5.0 User's guide", Systems Optimization Laboratory Dept. of Operations Research, Stanford University, Tech. Rep. (83-20), 1983.

[7] A. B. Philpott and G. Pritchard, "Financial transmission rights in convex pool markets", Operations Research Letters, vol. 32, pp. 109-113, 2004.

[8] M. S. Bazaraa, H. D. Sherali and C. M. Shetty, Nonlinear Programming, Theory and Algorithms, New York: Wiley, 2006.

[9] K. T. Chaturvedi , M. Pandit and L. Srivastava, "Self-organizing hierarchical particle swarm, optimization for nonconvex economic dispatch", IEEE Trans. on Power Syst., vol. 23, pp. 1079-1087, Aug. 2008.

[10] T. Alvey, D. Ma X. Goodwin and D. Sun, "A security-constrained bidclearing system for the New Zealand wholesale electricity market", IEEE Trans. on Power Syst., vol. 13, pp. 340-346, May 1998.

[11] S. De la Torre and F. Galiana, "On the Convexity of the System Loss Function", IEEE Trans. on Power Syst., vol. 20, pp. 2061-2069, Nov. 2005.

Rodrigo Palma-Behnke, (SM'04) received his B.Sc. and M.Sc. on Electrical Engineering from the Pontificia Universidad Católica de Chile and Dr.-Ing. from the University of Dortmund, Germany. $\mathrm{He}$ is currently professor in the Electrical Engineering Department at the University of Chile. His research field is the planning and operation of electrical systems in competitive power markets and new technologies.

Andy Philpott, (M'04) is a Professor in the Department of Engineering Science and leads the Electric Power Optimization Center at The University of Auckland, Auckland, New Zealand. His research interests encompass optimization under uncertainty, with particular reference to optimization modelling in electricity markets.

Alejandro Jofré, received the Mathematical Engineering diploma in 1984 from Universidad de Chile, his Ph.D. in Applied Mathematics (1989) from University of Pau, France and Habilitation Degree (1995) in France. He is currently a professor at Department of Mathematical at the University of Chile.

Marcelo Cortés-Carmona, (GS'07) received his B.Sc. in Electrical Engineering in 1989 from University of Antofagasta, Chile and his M.Sc. from University of Chile in 2005. He is currently a professor in the Electrical Engineering Department at the University of Antofagasta. He is currently pursuing the Ph.D. degree at the University of Chile. 